\title{
ON BOUNDARIES OF MODULI SPACES OF NON-SINGULAR CUBIC SURFACES WITH STAR POINTS
}

\author{
Nguyen Chanh Tu
}

\begin{abstract}
Let $\boldsymbol{P}^{19}$ be the parametrizing space of cubic surfaces in $\boldsymbol{P}^{3}$. Let $M$ be the moduli space of non-singular cubic surfaces and $\bar{M}$ be a suitable compactification. We study components of boundaries of the relative subspaces of non-singular cubic surfaces with star points in $\boldsymbol{P}^{19}$ and in $\bar{M}$.
\end{abstract}

\section{Introduction}

1.1. Let $\boldsymbol{P}^{19}$ be the parametrizing space of cubic surfaces in $\boldsymbol{P}_{k}^{3}$, where $k$ is an algebraically closed field with characteristic 0 . A hyperplane is called a tritangent plane with respect to a given cubic surface $X$ if the intersection consists of lines. A star point of $X$ is a common point of all lines of the intersection of a tritangent plane and $X$. If a smooth cubic surface $X$ has a star point then the corresponding hyperplane intersection consists of 3 distinct lines. This triple of lines is called a star triple. We denote $H_{s}$ for the subset of $\boldsymbol{P}^{19}$ corresponding to nonsingular cubic surfaces with at least $s$ star points. For each $H_{s}$, there is a decomposition into irreducible components $H_{s}^{(r)}$ where each $H_{s}^{(r)}$ in fact, corresponds to cubic surfaces generically containing exactly $r$ star points. Definitions of all $H_{s}^{(r)}$ together results on their irreducibility, dimensions and inclusion relationships could be found in [12] or [13].

1.2. We consider the action of $\operatorname{PGL}(4)$ on $\boldsymbol{P}^{19}$. Let $\phi:\left(\boldsymbol{P}^{19}\right)^{s s} \rightarrow \bar{M}$ be the quotient space with respect to the action of PGL(4) on $\boldsymbol{P}^{19}$, where $\left(\boldsymbol{P}^{19}\right)^{s s}$ is the subset of semi-stable points in the sense of geometric invariant theory (see [8], [9] or [11]). Let $M:=\operatorname{PGL}(4) \backslash\left(\boldsymbol{P}^{19}-\Delta\right)$, where $\Delta$ is the locus of singular cubic surfaces in $\boldsymbol{P}^{19}$. In fact, the space $\bar{M}$ is projective, and $M$ is the coarse moduli

2000 Mathematics Subject Classification: Primary 14D20, 14J10; Secondary 14J25.

Keywords and phrases: Varieties and morphisms, geometric invariant theory, special surfaces, embedding, moduli theory.

The author is supported by Japan Society for the Promotion of Science (JSPS) Postdoctoral Fellowship.

Received November 21, 2002; revised September 8, 2003. 
space of non-singular cubic surfaces (see [9], [10] or Section 3.2 of [13] for proofs). The space $\bar{M}$ is a compactification of $M$. Let $N:=\operatorname{PGL}(4) \backslash\left(\boldsymbol{P}^{19}\right)^{s}$, where $\left(\boldsymbol{P}^{19}\right)^{s}$ is the subset of stable points.

Let $(\Delta)^{s s}=\left(\boldsymbol{P}^{19}\right)^{s s} \cap \Delta$. We denote by $\Delta H_{s}^{(r)}$ the intersection of the closure of $H_{s}^{(r)}$ with $(\Delta)^{s s}$, which is called the boundary of $H_{s}^{(r)}$ in $\left(\boldsymbol{P}^{19}\right)^{s s}$. The corresponding space $\phi\left(\Delta H_{s}^{(r)}\right)$ is called the boundary of $H_{s}^{(r)}$ in $\bar{M}$.

1.3. We denote by $i \mathscr{A}_{1} j \mathscr{A}_{2}$ the subset of $\boldsymbol{P}^{19}$ corresponding to irreducible cubic surfaces with exactly $i$ singular points of type $A_{1}$ and $j$ singular points of type $A_{2}$. We refer to [1] and [2] or to [4] for general definitions of types of singularities. We use $j \mathscr{A}_{2}$ and $i \mathscr{A}_{1}$ instead of $0 \mathscr{A}_{1} j \mathscr{A}_{2}$ and $i \mathscr{A}_{1} 0 \mathscr{A}_{2}$ respectively. In fact, we have $2 i+3 j \leq 9, i \leq 4$ and $(i, j) \neq(3,1)$. It is well-known that $\left(\boldsymbol{P}^{19}\right)^{S S}$ (respectively $\left.\left(\boldsymbol{P}^{19}\right)^{s}\right)$ consists of $\boldsymbol{P}^{19}-\Delta$ and all of $i \mathscr{A}_{1} j \mathscr{A}_{2}$ (respectively $i \mathscr{A}_{1}$ ) (see [9], p. 51 or [13], 3.2.14 for a proof). We use the notation $\overline{i \mathscr{A}_{1} j \mathscr{A}_{2}}$ for the closure of $i \mathscr{A}_{1} j \mathscr{A}_{2}$ in $\left(\boldsymbol{P}^{19}\right)^{s s}$.

1.4. A singular, semi-stable cubic surface can be given by a polynomial in the following form:

$$
F=x_{3} f_{2}\left(x_{0}, x_{1}, x_{2}\right)+f_{3}\left(x_{0}, x_{1}, x_{2}\right),
$$

where $f_{i}$ for $i=1,2$ is a homogeneous polynomial of degree $i$ and the point $(0: 0: 0: 1)$ is a singular point. The type of singularity of the surface is characterized by $\operatorname{rank}\left(f_{2}\right)$ and the configuration of points in $V_{\boldsymbol{P}^{2}}\left(f_{2}, f_{3}\right)$ (see [4] for details). A closed subscheme in $\boldsymbol{P}^{2}$ of dimension 0 and of length 6 is called a 6-point scheme. Note that $V_{\boldsymbol{P}^{2}}\left(f_{2}, f_{3}\right)$ is a 6-point scheme with special configurations. Conversely, let $\mathscr{P}$ be a 6-point scheme in $\boldsymbol{P}^{2}$ with one of such configurations. Let $\mathscr{L}_{\mathscr{P}}$ be the linear space of cubic forms in $\boldsymbol{P}^{2}$ containing $\mathscr{P}$. Then $\mathscr{L}_{\mathscr{P}}$ has linear dimension 4 . Let $\left\{f_{1}, \ldots, f_{4}\right\}$ be a basis of $\mathscr{L}_{\mathscr{P}}$. Consider the morphism

$$
\begin{aligned}
\psi: \quad \boldsymbol{P}^{2}-\mathscr{P} & \rightarrow \boldsymbol{P}^{3} \\
P & \mapsto\left(f_{1}(P): f_{2}(P): f_{3}(P): f_{4}(P)\right) .
\end{aligned}
$$

Let $X$ be the closure of the image of $\psi$. Then $X$ is a semi-stable cubic surface. The surface $X$ is determined uniquely by $\mathscr{P}$ up to projective transformations. The surface $X$ is called the csurface of $\mathscr{P}$. In the case $\mathscr{P}$ consists of 6 points in general position, the surface $X$ is nothing but the blowing-up of $\boldsymbol{P}^{2}$ at the 6 points. We denote $c(\mathscr{P})$ for the formal cycle of a given 6-point scheme $\mathscr{P}$. We denote $S_{i j}$ for the two-dimensional linear subspace consisting of all cubic forms factoring into the linear form defining $l_{i j}=\bar{P}_{i} P_{j}$ and quadratic form passing through $\mathscr{P}_{-}\left\{P_{i}, P_{j}\right\}$. This subspace determines uniquely a line on $X$ which is denoted by $\tilde{l}_{i j}$. The line $\tilde{l}_{i j}$ is the closure of the image of $l_{i j}-\left\{P_{i}, P_{j}\right\}$. Similarly, we denote $S_{P_{i}}$ for the two-dimensional linear subspace consisting of cubic forms singular at $P_{i}$. This determines uniquely a line on $X$ which we denote by $\tilde{P}_{i}$. Also $S_{C_{i}}$ is denoted for the two-dimensional linear subspace consisting of all 
cubic forms factoring into the quadratic form defining the conic $C_{i}$ through $\left\{P_{1}, \ldots, P_{6}\right\}-\left\{P_{i}\right\}$ and linear form vanishing at $P_{i}$. This subspace determines uniquely a line on $X$, which is denoted by $\tilde{C}_{i}$. The line $\tilde{C}_{i}$ is nothing but the closure of the image of $C_{i}-\left\{P_{1}, P_{2}, P_{3}\right\}$.

We complete this section with two well-known results.

Lemma 1.1. The subsets $i \mathscr{A}_{1} j A_{2}$ are irreducible in $\boldsymbol{P}^{19}$.

Proof. This follows from [3]. Also see [13], 3.4.1.

Lemma 1.2. Let $x, y \in\left(\boldsymbol{P}^{19}\right)^{\text {ss }}$ such that the corresponding cubic surfaces contain one singular point of type $A_{2}$. Let $\phi:\left(\boldsymbol{P}^{19}\right)^{s S} \rightarrow \bar{M}$ be the quotient space. Then $\phi(x)=\phi(y)$. Consequently, the set $\bar{M}-N$ consists of the singleton s, which is the image of all non-stable points.

Remark 1.3. This was pointed out (without proof) in the introduction part of [10]. The reader could find a proof in [13], 3.4.2, which is formulated from a suggestion of Prof. Dr. E. Looijenga.

\section{On the boundary of $H_{1}$}

Recall that $H_{1}$ is the subvariety of $\boldsymbol{P}^{19}-\Delta$ parameterizing all cubic surfaces with at least one star point. The subset $H_{1}$ is irreducible of codimension 1 (see [12], p. 288).

A surface $X$ corresponding to a point in $2 \mathscr{A}_{1}$ has exactly 16 lines. There is one line on $X$ containing two singular points and the unique star point of $X$. See [13], p. 71 for the configuration of 16 lines of $X$.

Proposition 2.1. The subset $2 \mathscr{A}_{1}$ is contained in the closure of $H_{1}$. Consequently, the subset $\overline{2 \mathscr{A}_{1}}$ is a component of $\Delta\left(H_{1}\right)$. Moreover, the star point of $2 \mathscr{A}_{1}$ is the specialization position of star points on surfaces corresponding to points in $H_{1}$.

Proof. Let $x \in 2 \mathscr{A}_{1}$. From [4] or [13], p. 71, we see that the corresponding cubic surface $X_{x}$ is isomorphic to the csurface of a 6-point scheme $\mathscr{Q}=\sum_{i=1}^{6} Q_{i}$ where 3 points $Q_{2}, Q_{3}, Q_{6}$ lie on a line $l_{1}$; three points $Q_{4}, Q_{5}, Q_{6}$ lie on another line $l_{2}$; no 3 of the five points $Q_{1}, \ldots, Q_{5}$ are collinear (FIGURE 1).

Let $P_{t}$ be a moving point on the line $d=\overline{Q_{1} Q_{6}}$. At a general position of $P_{t}$ on $d$, the 6-point scheme $\mathscr{P}_{t}=\sum_{i=1}^{6} P_{i}$ where $P_{i}=Q_{i}$ for $1 \leq i \leq 5$ and $P_{6}=P_{t}$, gives a non-singular cubic surface with at least one star point. Except for a finite number of positions, when $P_{t}$ moves on the line $d$, we have a family in $H_{1}$. This implies that $x$ lies on the closure of $H_{1}$. Moreover, we see that the section of star points over the family is defined by the tritangent planes $H_{t}=\left(\tilde{l}_{23}, \tilde{l}_{45}, \tilde{l}_{16}\right)$. In the specialization position, the linear subspaces $S_{23}, S_{45}, S_{C_{1}}$ and $S_{Q_{6}}$ coincide. This means that $\tilde{Q}_{6}$ is the line connecting the 2 singular points and the section 


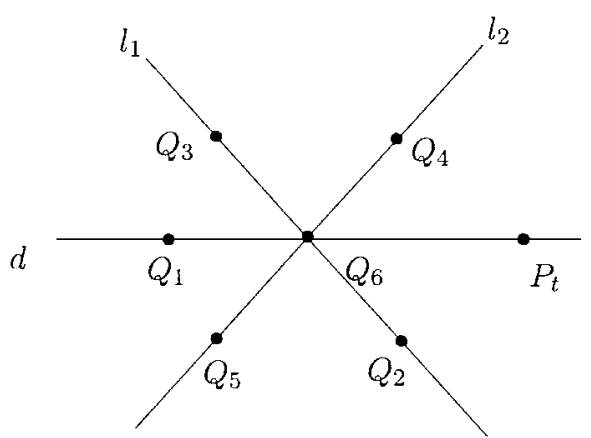

FIGURE 1. 6-point schemes giving points in $2 \mathscr{A}_{1}$

of tritangent planes $H_{t}$ contains the triple $\left(2 \tilde{Q}_{1}, \tilde{l}_{16}\right)$. This implies the last conclusion.

Recall that each surface corresponding to a point in $\mathscr{A}_{2}$ has exactly 15 lines ([4], p. 255). Moreover, any singular point of type $A_{2}$ is a star point ([13], p. 82).

Proposition 2.2. Any $x \in \mathscr{A}_{2}$ lies on the closure of $H_{1}$. Consequently, the subset $\overline{\mathscr{A}}_{2}$ is a component of $\Delta\left(H_{1}\right)$. Moreover, the $A_{2}$ singularity of $X_{x}$, as a star point, is the specialization position of star points on surfaces corresponding to points in $H_{1}$.

Proof. Let $\mathscr{Q}$ be a 6-point scheme where $c(\mathscr{Q})=2 Q_{1}+\sum_{i=2}^{5} Q_{i}$, such that three points $Q_{1}, Q_{2}, Q_{3}$ are contained in a line $l$; the direction at double point $2 Q_{1}$ does not contain any $Q_{i}$ for $i=2,3,4,5$; the four points $Q_{1}, Q_{2}, Q_{4}, Q_{5}$ as well as 4 points $Q_{1}, Q_{3}, Q_{4}, Q_{5}$ are in general position (FIGURE 2, (a)). We know that ([13], p. 73 and p. 86), the csurface of $\mathscr{P}$ is isomorphic to a cubic surface with exactly one $A_{2}$ singularity.

Let $x \in \mathscr{A}_{2}$. The surface $X_{x}$ is isomorphic to the csurface of a 6-point scheme $\mathscr{Q}$ where $c(\mathscr{Q})=2 Q_{1}+\sum_{i=2}^{5} Q_{i}$ described as above.

Let $O$ be the intersection point of $l$ and $\overline{Q_{4} Q_{5}}$. Let $d$ be the direction at the double point $2 Q_{1}$. Let $m$ be a fixed line which contains $Q_{3}$ and does not contain any other point in $\left\{Q_{1}, \ldots, Q_{5}\right\}$. Let $\left(P_{6}, P_{3}\right)$ be a pair of moving points where $P_{6} \in d$ and $P_{3} \in m$ such that $P_{3} P_{6}$ contains $O$. It is clear that, except for a finite number of positions, when moving $\left(P_{6}, P_{3}\right)$, the csurfaces of 6-point schemes $\mathscr{P}=\sum_{i=1}^{6} P_{i}$, where $P_{i}=Q_{i}$ for $i \in\{1,2,4,5\}$, are isomorphic to non-singular cubic surfaces with at least one star point. This defines a family in $H_{1}$. When $\left(P_{6}, P_{3}\right)=\left(Q_{1}, Q_{3}\right)$, we get 6-point scheme 2 whose csurface is isomorphic to $X_{x}$. So $x$ lies on the closure of $H_{1}$. Moreover, the star section over the family is defined by the tritangent planes $\left(\tilde{l}_{12}, \tilde{l}_{45}, \tilde{l}_{36}\right)$. In the specialization position, the linear subspaces $S_{12}, S_{26}$ and $S_{Q_{3}}$ coincide; the linear subspaces $S_{36}, S_{13}$ and $S_{Q_{2}}$ 

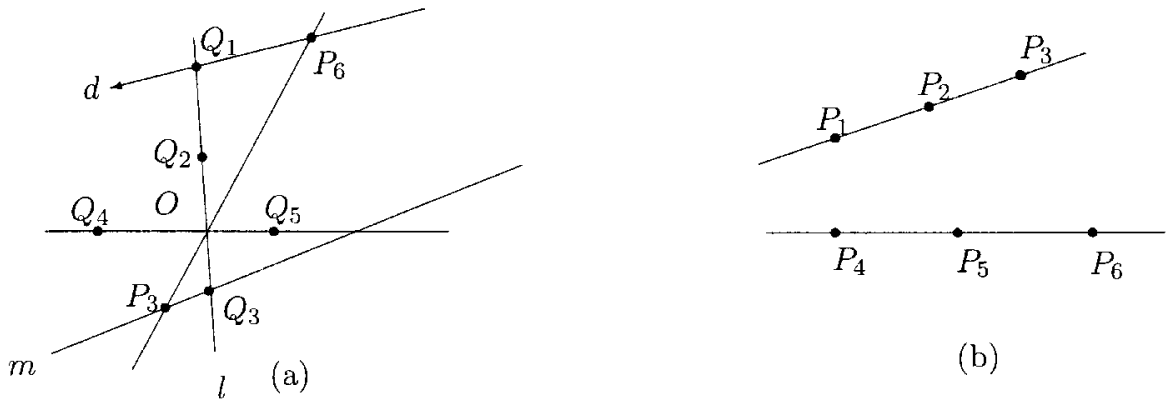

(b)

FIGURE 2. 6-point schemes giving points in $\mathscr{A}_{2}$

coincide. Note that the 6 lines $\tilde{Q}_{1}, \tilde{Q}_{2}, \tilde{Q}_{3}, \tilde{l}_{45}, \tilde{l}_{14}$ and $\tilde{l}_{15}$ contain the $A_{2}$ singularity. It is clear that the section of star points gives a specialization to the intersection of $\tilde{Q}_{2}, \tilde{Q}_{3}$ and $\tilde{l}_{45}$, which is the $A_{2}$ singularity.

Remark 2.3. In [13], p. 82, there is a list of all star points on semi-stable cubic surfaces. Moreover, there is a definition of proper star point, which is the specialization position of some section of star points on non-singular cubic surfaces. In fact, all star points on semi-stable cubic surfaces are proper star points ([13], 3.4.10).

Consider the set $K_{1}$ consisting of all 6-point schemes $\mathscr{P}$ where $c(\mathscr{P})=\sum_{i=1}^{6} P_{i}$ such that $\mathscr{P}$ is contained in an irreducible conic and $l_{12} \cap l_{34} \cap l_{56}=\{O\}$ (FIGURE $3)$.

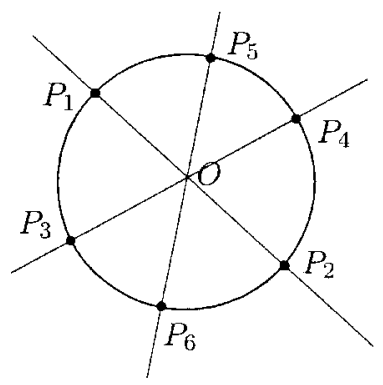

Figure 3. 6-point schemes of $K_{1}$

Let $D_{1}$ be the subset of $\boldsymbol{P}^{19}$ consisting of all points corresponding to the cubic surfaces, each of them is isomorphic to the csurface of an element in $K_{1}$. It is clear that $D_{1} \subset \mathscr{A}_{1}$. We see that $D_{1}$ is contained in the closure of $H_{1}$ also. For this, let $x \in D_{1}$. The corresponding surface $X_{x}$ is isomorphic to the csurface of some 6-point scheme $\mathscr{P} \in K$. Let $c(\mathscr{P})=\sum_{i=1}^{6} P_{i}$. Fix $P_{1}, \ldots, P_{5}$ and let $P_{6}$ 
move on the line $\overline{P_{5} O}$. This defines a family of 6-point schemes whose csurfaces are isomorphic to non-singular cubic surfaces with at least one star point. When $P_{6}$ is contained in the conic defined by $P_{1}, \ldots, P_{5}$, we get $\mathscr{P}$. This implies that $x$ lies on the closure of $H_{1}$. Moreover, we prove that:

Lemma 2.4. The subset $D_{1}$ is irreducible in $\left(\boldsymbol{P}^{19}\right)^{s s}$.

Proof. Let $x \in D_{1}$. Since $D_{1} \subset \mathscr{A}_{1}$, by choosing coordinates, we can assume that $x$ is given by

$$
F=x_{3}\left(x_{1}^{2}-x_{0} x_{2}\right)+f_{3}
$$

where

$$
f_{3}=a_{0} x_{0}^{3}+a_{1} x_{0}^{2} x_{1}+a_{2} x_{0} x_{1}^{2}+a_{3} x_{1}^{3}+a_{4} x_{1}^{2} x_{2}+a_{5} x_{1} x_{2}^{2}+a_{6} x_{2}^{2},
$$

such that the scheme $V_{\boldsymbol{P}^{2}}\left(x_{1}^{2}-x_{0} x_{2}, f_{3}\right)$ consists of 6 distinct points (see [4], pp. 247-248). Furthermore, there exists a numbering of 6 points $P_{1}, \ldots, P_{6}$ of $V_{\boldsymbol{P}^{2}}\left(x_{1}^{2}-x_{0} x_{2}, f_{3}\right)$ such that $l_{12} \cap l_{34} \cap l_{56} \neq \emptyset$ (FIGURE 3$)$.

The 6 points of $V_{P^{2}}\left(x_{1}^{2}-x_{0} x_{2}, f_{3}\right)$ are determined by the solutions of the equation

$$
a_{0} \theta^{6}+a_{1} \theta^{5} \psi+a_{2} \theta^{4} \psi^{2}+a_{3} \theta^{3} \psi^{3}+a_{4} \theta^{2} \psi^{4}+a_{5} \theta \psi^{5}+a_{6} \psi^{6}=0 .
$$

Let $T$ be the projective space parameterizing all homogeneous polynomials of degree 6 in two variables.

Consider the morphism

$$
\begin{aligned}
\left(\boldsymbol{P}^{1}\right)^{6} & \rightarrow T \\
\left(a_{1}: b_{1} ; \cdots ; a_{6}: b_{6}\right) & \mapsto \prod_{i=1}^{6}\left(b_{i} \theta-a_{i} \psi\right) .
\end{aligned}
$$

Each solution $\left(\theta_{i}: \psi_{i}\right)$ of (2) corresponds to a point $P_{i}=\left(\theta_{i}^{2}: \theta_{i} \psi_{i}: \psi_{i}^{2}\right)$ for $1 \leq i \leq 6$ which is contained in the conic $V_{\boldsymbol{P}^{2}}\left(x_{1}^{2}-x_{0} x_{2}\right)$. We see that, the set of all elements of $\left(\boldsymbol{P}^{1}\right)^{6}-\Delta$ such that $l_{12} \cap l_{34} \cap l_{56} \neq \emptyset$ is irreducible. This implies that the subset $D_{1}^{\prime} \subset \boldsymbol{P}^{19}$ which consists of all elements corresponding to polynomials of the form (1) and satisfying the above condition is irreducible. We see that $D_{1}$ is the image of the morphism $\varphi: \operatorname{PGL}(4) \times D_{1}^{\prime} \rightarrow \boldsymbol{P}^{19}$ which is induced from the action of PGL(4) on $\boldsymbol{P}^{19}$. So the set $D_{1}$ is irreducible.

THEOREM 2.5. $\Delta H_{1}=\overline{D_{1}} \cup \overline{2 \mathscr{A}_{1}} \cup \overline{\mathscr{A}_{2}}$.

Proof. It is clear that the sets $\overline{D_{1}}, \overline{\mathscr{A}_{1}}$ and $\overline{\mathscr{A}_{2}}$ are irreducible components of $\Delta H_{1}$. Conversely, let $x$ be the generic point of an irreducible component $W$ of $\Delta H_{1}$. Suppose that $W \neq \overline{2 \mathscr{A}_{1}}$ and $W \neq \overline{\mathscr{A}_{2}}$. Since $\operatorname{dim} W=17$, we have $x \in \mathscr{A}_{1}$. So the surface $X_{x}$ is isomorphic to the csurface of a 6-point scheme $\mathscr{P}$ such that $c(\mathscr{P})=\sum_{i=1}^{6} P_{i}$ where 6 distinct points $P_{1}, \ldots, P_{6}$ are contained in 
an irreducible conic. Note that $X_{x}$ has exactly 21 lines (see [4], p. 249). The 21 lines of $X_{x}$ are $\tilde{P}_{i}$ and $\tilde{l}_{i j}$ for $1 \leq i<j \leq 6$. Note that any singular point of type $A_{1}$ is not a star point (see [13], p. 82). Therefore, the star point of $X_{x}$ is determined by a triple of the form $\left(\tilde{l}_{i j}, \tilde{l}_{h k}, \tilde{l}_{m n}\right)$. This implies that the 6 points $P_{1}, \ldots, P_{6}$ satisfy $l_{i j} \cap l_{h k} \cap l_{m n} \neq \emptyset$. This means that $x \in D_{1}$ and $W=\bar{D}_{1}$.

Recall that $\phi:\left(\boldsymbol{P}^{19}\right)^{S S} \rightarrow \bar{M}$ is the quotient space with respect to the action of PGL(4) on $\boldsymbol{P}^{19}$.

COROLlaRy 2.6. $\phi\left(\Delta H_{1}\right)=\phi\left(\overline{D_{1}}\right) \cup \phi\left(\overline{2 \mathscr{A}_{1}}\right)$. Moreover, the components $\phi\left(\overline{D_{1}}\right)$ and $\phi\left(\overline{2 \mathscr{A}_{1}}\right)$ contain the singleton $s$.

Proof. Since $\phi:\left(\boldsymbol{P}^{19}\right)^{s s} \rightarrow \bar{M}$ is a good quotient (see [11], 2.13 or [13], $3.2 .8)$, the sets $\phi\left(\overline{D_{1}}\right)$ and $\phi\left(\overline{2 \mathscr{A}_{1}}\right)$ are closed. Moreover, since $\overline{2 \mathscr{A}_{2}} \subset \overline{2 \mathscr{A}_{1}}$, we have $s \in \phi\left(\overline{2 \mathscr{A}_{1}}\right)$. The first conclusion follows from the theorem.

Let $\mathscr{P} \in K$. By definition, we have $c(\mathscr{P})=\sum_{i=1}^{6} P_{i}$ where 6 points $P_{1}, \ldots, P_{6}$ are contained in an irreducible conic and $l_{12} \cap l_{34} \cap l_{56}=\{O\}$. Consider the quadratic transformation $\varphi$ with respect to $P_{1}, P_{3}$ and $P_{5}$, we see that the 6-point scheme $\varphi(\mathscr{P})$ consists of 6 distinct points $Q_{1}, \ldots, Q_{6}$ such that $Q_{2}, Q_{4}, Q_{6}$ are collinear and $\overline{Q_{1} Q_{2}} \cap \overline{Q_{3} Q_{4}} \cap \overline{Q_{5} Q_{6}}=\{\varphi(O)\}$ (FIGURE 4). The 6-point scheme $\varphi(\mathscr{P})$ corresponds to a csurface in $D_{1}$ (see [12], 3.3.10).

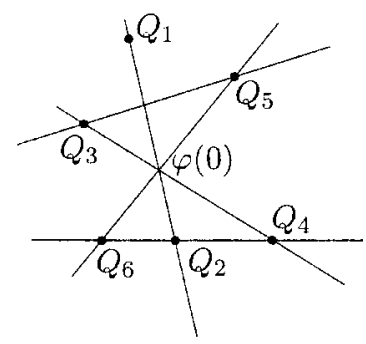

FIGURE 4. 6-point schemes giving points in $D_{1}$

Consider a family in $D_{1}$ given by fixing 5 points $Q_{2}, \ldots, Q_{6}$ and moving $Q_{1}$ on the line $\overline{Q_{2} \varphi(O)}$, where $\varphi(O)=\overline{Q_{3} Q_{4}} \cap \overline{Q_{5} Q_{6}}$. When $Q_{1}$ coincides with the intersection point of $\overline{Q_{2} \varphi(O)}$ and $\overline{Q_{3} Q_{5}}$, we get a 6-point scheme whose csurface is isomorphic to a surface with exactly one $A_{2}$ singularity. This implies that $s \in \phi\left(\overline{D_{1}}\right)$.

\section{On the boundaries of $H_{2}^{(2)}$ and $H_{2}^{(3)}$}

Recall that $H_{2}$ is the subset of $\boldsymbol{P}^{19}-\Delta$ corresponding to non-singular cubic surfaces with at least two star points. The space $\mathrm{H}_{2}$ is of codimension 2 and has two irreducible components $H_{2}^{(2)}$ and $H_{2}^{(3)}$. The subset $H_{2}^{(2)}$ parametrizes non- 
singular cubic surfaces containing two star triples with one line in common. The subset $H_{2}^{(3)}$ parametrizes non-singular cubic surfaces containing two star triples with no line in common; these surfaces have at least 3 star points, which are collinear (see [12], p. 289 or [13], 2.3.1).

\subsection{On the boundary of $H_{2}^{(3)}$}

Proposition 3.1. The set $3 \mathscr{A}_{1}$ is contained in the closure of $\mathrm{H}_{2}^{(3)}$. Consequently, the set $\overline{3 \mathscr{A}_{1}}$ is an irreducible component of $\Delta H_{2}^{(3)}$.

Proof. Let $x \in 3 \mathscr{A}_{1}$. The corresponding surface $X_{x}$ can be considered as the csurface of a 6-point scheme 2 consisting of 6 distinct points $Q_{1}, \ldots, Q_{6}$ such that $Q_{1}, Q_{3}, Q_{6}$ as well as $Q_{3}, Q_{5}, Q_{2}$ and $Q_{4}, Q_{5}, Q_{6}$ are collinear; moreover $Q_{1} \notin \overline{Q_{2} Q_{4}}$ (FIgURE 5). See [13], p. 74 for other configurations of $3 \mathscr{A}_{1}$.

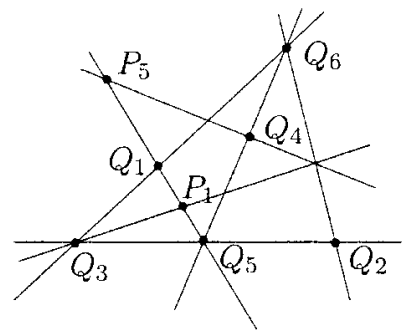

FIGURE 5. 6-point schemes giving points in $3 \mathscr{A}_{1}$

Consider a family of 6-point schemes $\mathscr{P}=\sum_{i=1}^{6} P_{i}$ where $P_{i}=Q_{i}$ for $i \in$ $\{2,3,4,6\}$ and $P_{1}, P_{5}$ move on the line $\overline{Q_{1} Q_{5}}$ in such a way that $\overline{P_{1} Q_{3}} \cap \overline{Q_{4} P_{5}} \cap$ $\overline{Q_{2} Q_{6}} \neq \emptyset$. Except for a finite number of positions, each pair $\left(P_{1}, P_{5}\right)$ gives a 6-point scheme such that its csurface is isomorphic to a cubic surface in $H_{2}^{(3)}$. This gives a family in $H_{2}^{(3)}$. The three star points are given by $\left(\tilde{l}_{15}, \tilde{l}_{23}, \tilde{l}_{46}\right)$, $\left(\tilde{l}_{13}, \tilde{l}_{45}, \tilde{l}_{26}\right)$ and $\left(\tilde{l}_{34}, \tilde{l}_{16}, \tilde{l}_{25}\right)$. When $P_{1}=Q_{1}$ then $P_{5}=Q_{5}$. This implies that $x \in \Delta H_{2}^{(3)}$.

Definition. Let $K_{0}$ be the subset of $\left(\boldsymbol{P}^{2}\right)^{4}$ consisting of 4-tuple $\left(P_{1}, P_{2}, P_{3}\right.$, $\left.P_{4}\right)$ in general position. Let

$$
\begin{aligned}
K_{1}=\{( & \left.P_{1}, P_{2}, P_{3}, P_{4}, P_{5}\right) \in\left(\boldsymbol{P}^{2}\right)^{5} \mid\left(P_{1}, P_{2}, P_{3}, P_{4}\right) \in K_{0} ; P_{5} \in l_{12} ; P_{5} \notin l_{34} ; \\
P_{5} \neq & \left.P_{i}, \forall i=1,2\right\} ; \\
B_{2}^{(3)}= & \left\{\left(P_{1}, P_{2}, P_{3}, P_{4}, P_{5}, P_{6}\right) \in\left(\boldsymbol{P}^{2}\right)^{6} \mid\left(P_{1}, P_{2}, P_{3}, P_{4}, P_{5}\right) \in K_{1} ;\right. \\
& \left.l_{16} \cap l_{24} \cap l_{35} \neq \emptyset ; l_{14} \cap l_{23} \cap l_{56} \neq \emptyset\right\} \quad \text { (FIgure 6). }
\end{aligned}
$$




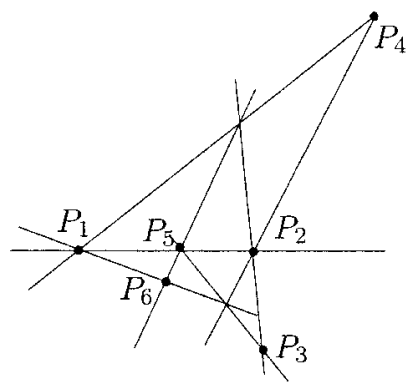

FIGURE 6. 6-point schemes giving points in $D_{2}^{(3)}$

Let $D_{2}^{(3)}$ be the subset of $\boldsymbol{P}^{19}$ consisting of all points such that each corresponding cubic surface is isomorphic to the csurface of a 6-point scheme determined by some 6 -tuple in $B_{2}^{(3)}$. It is easy to see that $D_{2}^{(3)} \subset \mathscr{A}_{1}$.

Proposition 3.2. The closure of $D_{2}^{(3)}$ is an irreducible component of $\Delta H_{2}^{(3)}$.

Proof. First of all, we prove that $D_{2}^{(3)}$ is irreducible. The set $K_{0}$ is an open subset of $\left(\boldsymbol{P}^{2}\right)^{4}$ so it is irreducible. Each fiber of the projection $p: K_{1} \rightarrow K_{0}$ is isomorphic to an open set of $\boldsymbol{P}^{1}$. This implies that $K_{1}$ is irreducible. Since $K_{1}$ is isomorphic to $B_{2}^{(3)}$, the set $B_{2}^{(3)}$ is irreducible.

Let

$$
\begin{gathered}
L=\left\{\left(\mathscr{P}, F_{1}, F_{2}, F_{3}, F_{4}\right) \mid \mathscr{P} \in B_{2}^{(3)} ; F_{i} \text { for } 1 \leq i \leq 4 \text { is a cubic form in } \mathscr{L}_{\mathscr{P}}\right\}, \\
U=\left\{\left(\mathscr{P}, F_{1}, F_{2}, F_{3}, F_{4}\right) \in L \mid\left\{F_{1}, F_{2}, F_{3}, F_{4}\right\} \text { is a basis of } \mathscr{L}_{\mathscr{P}}\right\} .
\end{gathered}
$$

Consider the following diagram:

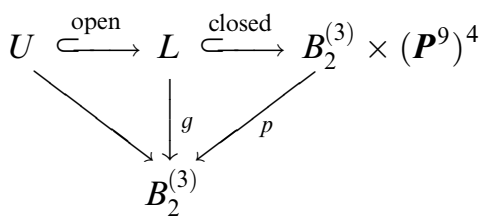

where $p$ is the projection. The map $g$ is surjective and every fiber is isomorphic to $\left(\boldsymbol{P}^{3}\right)^{4}$. So $L$ is irreducible. This implies that $U$ is irreducible.

Let $D_{1}$ be the subset of $B_{2}^{(3)} \times \boldsymbol{P}^{19}$ consisting of all pairs $(\mathscr{P}, x)$ where the cubic surface corresponding to $x$ is isomorphic to the csurface of the 6-point scheme determined by $\mathscr{P}$. Given $\left(\mathscr{P}, F_{1}, F_{2}, F_{3}, F_{4}\right) \in U$, the closure of the rational map from $\boldsymbol{P}^{2}$ to $\boldsymbol{P}^{3}$ defined by the basis $\left\{F_{1}, F_{2}, F_{3}, F_{4}\right\}$ is a cubic surface in $\boldsymbol{P}^{3}$. We have a morphism $\tau: U \rightarrow D_{1}$ which is surjective. This implies that $D_{1}$ is irreducible. The projection $D_{1} \rightarrow D_{2}^{(3)}$ is surjective. Consequently $D_{2}^{(3)}$ is irreducible.

Let $x \in D_{2}^{(3)}$. The corresponding surface $X_{x}$ can be considered as the csurface of a 6-point scheme $\mathscr{P}$ such that $c(\mathscr{P})=\sum_{i=1}^{6} P_{i}$ where $\left(P_{1}, \ldots, P_{6}\right) \in B_{2}^{(3)}$. 
Fix $P_{1}, P_{2}, P_{6}, P_{4}$ and let $P_{3}, P_{5}$ move on the lines $l_{23}, l_{56}$, respectively in such a way that $l_{16} \cap l_{24} \cap l_{35} \neq \emptyset$. We obtain a family in $H_{2}^{(3)}$ such that $x$ is a specialization position. In this family, the three star points are given by $\left(\tilde{l}_{14}, \tilde{l}_{23}, \tilde{l}_{56}\right),\left(\tilde{l}_{16}, \tilde{l}_{24}, \tilde{l}_{35}\right)$ and $\left(\tilde{l}_{26}, \tilde{l}_{45}, \tilde{l}_{13}\right)$. Moreover, we see that when $P_{5}=P_{6}$ then $P_{3}$ moves to the intersection point of $l_{16}$ and $l_{23}$. This gives a 6-point scheme whose csurface is isomorphic to a cubic surface with exactly one $A_{2}$ singularity (FIGURE 2, (a)).

Lemma 3.3. Let $x$ be the generic point of an irreducible component of $\Delta H_{2}^{(3)}$. Then $x \notin 2 \mathscr{A}_{1}$.

Proof. Suppose that $x \in 2 \mathscr{A}_{1}$. Let $S_{1}, S_{2}, S_{3}$ be the three star points of $X_{x}$. By choosing coordinates, we can assume that the corresponding cubic surface $X_{x}$ is isomorphic to the csurface of a 6-point scheme $\mathscr{Q}$ such that $c(\mathscr{Q})=\sum_{i=1}^{6} Q_{i}$ where $Q_{6}, Q_{2}, Q_{3}$ as well as $Q_{6}, Q_{4}, Q_{5}$ are collinear, and no 3 of the five points $Q_{1}, \ldots, Q_{5}$ are collinear (see FIgURE 1 ). The line $\tilde{Q}_{6}$ contains the two singular points and has multiplicity 4 . The line $\tilde{l}_{16}$ intersects $\tilde{Q}_{6}$ but does not contain any singular point. Note that the 9 lines of the 3 star triples of a non-singular cubic surface in $H_{2}^{(3)}$ are mutually different. Since the line $\tilde{l}_{16}$ has multiplicity 1 , the line $\tilde{l}_{16}$ does not contain all $S_{1}, S_{2}, S_{3}$. Thus there exists a star triple whose lines are different from $\tilde{l}_{16}$. But from the configuration of lines and tritangent planes together their multiplicities (see [6], Articles 35-201 or [13], pp. 71-72), we see that there does not exist a such star triple.

TheORem 3.4. Let $\phi:\left(\boldsymbol{P}^{19}\right)^{s s} \rightarrow \bar{M}$ be the quotient space with respect to the action of $\operatorname{PGL}(4)$ on $\boldsymbol{P}^{19}$. Then $\phi\left(\Delta H_{2}^{(3)}\right)=\phi\left(\overline{D_{2}^{(3)}}\right) \cup \phi\left(\overline{\overline{3 \mathscr{A}}_{1}}\right)$. Moreover, the components $\phi\left(\overline{D_{2}^{(3)}}\right)$ and $\phi\left(\overline{3 \mathscr{A}_{1}}\right)$ contain the singleton $s$.

Proof. By the end of the proof of Proposition 3.2, we see that the boundary of $D_{2}^{(3)}$ contains a point of $\mathscr{A}_{2}$. So $s \in \phi\left(\overline{D_{2}^{3}}\right)$. Since $3 \mathscr{A}_{2} \subset \overline{3 \mathscr{A}_{1}}$, we have $s \in \phi\left(\overline{3 \mathscr{A}_{1}}\right)$.

Let $x$ be the generic point of an irreducible component $W$ of $\phi\left(\Delta H_{2}^{(3)}\right)$. Suppose that $W \neq \phi\left(\overline{3 \mathscr{A}_{1}}\right)$. From the previous lemma, we see that $x \notin 2 \mathscr{A}_{1}$. Therefore $x \in \mathscr{A}_{1}$. By choosing coordinates, we can assume that the surface $X_{x}$ is isomorphic to the csurface of a 6-point scheme $\mathscr{P}$ where $c(\mathscr{P})=\sum_{i=1}^{6} P_{i}$ such that the 6 points $P_{1}, \ldots, P_{6}$ are contained in an irreducible conic. Since the singular point of $X_{x}$ is not a star point, the 3 star points of $X_{x}$ are determined by 3 triples $\left(\tilde{l}_{i j}, \tilde{l}_{m n}, \tilde{l}_{k h}\right),\left(\tilde{l}_{i m}, \tilde{l}_{j k}, \tilde{l}_{n h}\right)$ and $\left(\tilde{l}_{m k}, \tilde{l}_{i h}, \tilde{l}_{j n}\right)$, where $\{i, j, m, n, h, k\}=\{1,2,3$, $4,5,6\}$. This implies that the 6 points $P_{1}, \ldots, P_{6}$ in $\boldsymbol{P}^{2}$ satisfy the corresponding conditions, namely $l_{i j} \cap l_{m n} \cap l_{k h} \neq \emptyset, l_{i m} \cap l_{j k} \cap l_{n h} \neq \emptyset$ and $l_{m k} \cap l_{i h} \cap l_{j n} \neq \emptyset$. Consider the quadratic transformation with respect to $P_{i}, P_{n}, P_{k}$ ([13], 3.3.10), we see that the image of $\mathscr{P}$ is a 6-point scheme $\mathscr{Q}$ where $c(\mathscr{Q})=\sum_{i=1}^{6} Q_{i}$ such that 6 points $Q_{1}, \ldots, Q_{6}$, up to a permutation of 6 letters, form an element of $B_{2}^{(3)}$. This implies that $W=\phi\left(\overline{D_{2}^{(3)}}\right)$. 


\subsection{On the boundary of $H_{2}^{(2)}$}

DEFINITION. Let

$$
\begin{aligned}
B_{2}^{(2)}=\{ & \left(P_{1}, \ldots, P_{5}, O\right) \in\left(\boldsymbol{P}^{2}\right)^{6} \mid P_{1}, P_{2}, P_{3}, P_{4}, P_{5} \text { are in general position; } \\
& l_{24} \cap l_{35}=\{O\} ; \text { the conic determined by } P_{1}, \ldots, P_{5} \text { is tangent } \\
& \text { to } \left.\overline{P_{1} O} \text { at } P_{1}\right\}(\text { Figure } 7 \text { (a)). }
\end{aligned}
$$

Note that each element in $B_{2}^{(2)}$ defines uniquely a 6-point scheme $\mathscr{P}$ such that $c(\mathscr{P})=2 P_{1}+\sum_{i=2}^{5} P_{i}$, where the direction at the double point $2 P_{1}$ is determined by $\overline{P_{1} O}$. Moreover, the csurface of $\mathscr{P}$ has exactly two $A_{1}$ singularities ([13], pp. $71-72)$. Let $D_{2}^{(2)}$ be the subset of $\boldsymbol{P}^{19}$ consisting of all points such that each corresponding cubic surface is isomorphic to the csurface of a 6-point scheme determined by some element of $B_{2}^{(2)}$. Then $D_{2}^{(2)} \subset 2 \mathscr{A}_{1}$.

Let

$$
\begin{aligned}
C_{2}^{(2)}=\{ & \left(P_{1}, \ldots, P_{6}\right) \in\left(\boldsymbol{P}^{2}\right)^{6} \mid P_{i} \neq P_{j} \forall i \neq j ; l_{12} \cap l_{34} \cap l_{56} \neq \emptyset ; l_{14} \cap l_{23} \cap l_{56} \neq \emptyset ; \\
& \left.P_{1}, P_{2}, P_{3}, P_{4}, P_{5}, P_{6} \text { are contained in an irreducible conic }\right\}
\end{aligned}
$$

(FiguRe 7 (b)).

Let $E_{2}^{(2)}$ be the subset of $\boldsymbol{P}^{19}$ consisting of all points such that each corresponding cubic surface is isomorphic to the csurface of a 6-point scheme determined by some 6-tuple in $C_{2}^{(2)}$. Then $E_{2}^{(2)} \subset \mathscr{A}_{1}$.

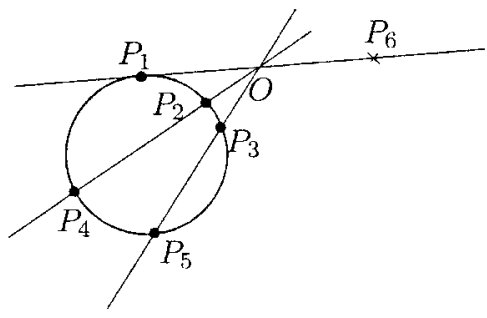

(a)

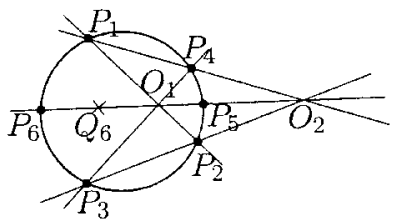

(b)

FIGURE 7. 6-point schemes of $B_{2}^{(2)}$ and $C_{2}^{(2)}$, respectively $\Delta H_{2}^{(2)}$.

Proof. Let $\boldsymbol{P}^{5}$ be the projective space parameterizing the non-zero quadratic forms in three variables. Let 


$$
\begin{aligned}
K_{0}= & \left\{\left(P_{1}, P_{2}, P_{3}, P_{4}\right) \in\left(\boldsymbol{P}^{2}\right)^{4} \mid P_{1}, P_{2}, P_{3}, P_{4} \text { are in general position }\right\} ; \\
K_{1}=\{ & \left(P_{1}, P_{2}, P_{3}, P_{4}, O, \mathscr{C}\right) \in\left(\boldsymbol{P}^{2}\right)^{5} \times \boldsymbol{P}^{5} \mid\left(P_{1}, P_{2}, P_{3}, P_{4}\right) \in K_{0} ; O \in l_{24} ; \\
& O \notin\left\{P_{2}, P_{4}\right\} ; O \notin l_{13} ; \mathscr{C} \text { is the conic containing } P_{1}, P_{2}, P_{3}, P_{4} \text { and } \\
& \text { tangent to } \left.\overline{P_{1} O} \text { at } P_{1}\right\} ; \\
K_{2}= & \left\{\left(P_{1}, P_{2}, P_{3}, P_{4}, P_{5}, O, \mathscr{C}\right) \in\left(\boldsymbol{P}^{2}\right)^{6} \times \boldsymbol{P}^{5} \mid\left(P_{1}, P_{2}, P_{3}, P_{4}, O, \mathscr{C}\right) \in K_{1} ;\right. \\
& \left.P_{5} \in \mathscr{C} \cap \overline{P_{3} O}\right\} .
\end{aligned}
$$

It is clear that the set $K_{0}$ is irreducible. Every fiber of the projection $p_{1}: K_{1} \rightarrow K_{0}$ is isomorphic to an open set of $\boldsymbol{P}^{1}$. This implies that $K_{1}$ is irreducible. The projections $K_{2} \rightarrow K_{1}$ and $K_{2} \rightarrow B_{2}^{(2)}$ are isomorphisms. Therefore $B_{2}^{(2)}$ is irreducible.

Similarly, we prove that $C_{2}^{(2)}$ is irreducible.

By a similar argument as used in the proof of (3.2), we see that $D_{2}^{(2)}$ and $E_{2}^{(2)}$ are irreducible.

Suppose $x \in D_{2}^{(2)}$. The corresponding surface $X_{x}$ is isomorphic to the csurface of a 6-point scheme $\mathscr{P}_{0}$ determined by an element $\left(P_{1}, \ldots, P_{5}, O\right) \in B_{2}^{(2)}$. Consider a family of $H_{2}^{(2)}$ given by 6-point schemes $\mathscr{P}$ such that $c(\mathscr{P})=\sum_{i=1}^{6} P_{i}$ where $P_{6}$ is a moving point on the line $\overline{P_{1} O}$ (FIgure 7 (a)). The two star points are given by $\left(\tilde{P}_{1}, \tilde{C}_{6}, \tilde{l}_{16}\right)$ and $\left(\tilde{l}_{16}, \tilde{l}_{24}, \tilde{l}_{35}\right)$. This implies that $x \in \Delta H_{2}^{(2)}$.

Let $x$ be an element in $E_{2}^{(2)}$. The cubic surface $X_{x}$ corresponding to $x$ is isomorphic to the csurface of a 6-point scheme $\mathscr{P}_{0}$ determined by a 6-tuple $\left(P_{1}, \ldots, P_{6}\right)$ in $C_{2}^{(2)}$. Consider a family of $H_{2}^{(2)}$ given by 6-point schemes $\mathscr{2}$ such that $c(\mathscr{Q})=\sum_{i=1}^{6} Q_{i}$ where $Q_{i}=P_{i}$ for $1 \leq i \leq 5$ and $Q_{6}$ is a moving point on the line $\overline{O_{1} O_{2}}($ FIGURE $7(\mathrm{~b}))$. The two star points are given by $\left(\tilde{l}_{12}, \tilde{l}_{34}, \tilde{l}_{56}\right)$ and $\left(\tilde{l}_{56}, \tilde{l}_{14}, \tilde{l}_{23}\right)$. This implies that $x \in \Delta H_{2}^{(2)}$.

Proposition 3.6. Let $x$ be the generic point of an irreducible component $W$ of $\Delta H_{2}^{(2)}$. If $x \in \mathscr{A}_{1}$, then $W=\overline{E_{2}^{(2)}}$.

Proof. By choosing coordinates, we can assume that the surface $X_{x}$ is isomorphic to the csurface of a 6-point scheme $\mathscr{P}$ such that $c(\mathscr{P})=\sum_{i=1}^{6} P_{i}$ where 6 points $P_{1}, \ldots, P_{6}$ are contained in an irreducible conic. Since the $A_{1}$ singularity is not a star point, the two star points of $X_{x}$ are defined by triples $\left(\tilde{l}_{i j}, \tilde{l}_{m n}, \tilde{l}_{k h}\right)$ and $\left(\tilde{l}_{i j}, \tilde{l}_{m k}, \tilde{l}_{n h}\right)$ where $\{i, j, m, n, h, k\}=\{1,2,3,4,5,6\}$. This implies that the 6 points $P_{1}, \ldots, P_{6}$ in $\boldsymbol{P}^{2}$ satisfy the corresponding conditions, namely $l_{i j} \cap l_{m n} \cap l_{k h} \neq \emptyset$ and $l_{i j} \cap l_{m k} \cap l_{n h} \neq \emptyset$. Up to a permutation of 6 letters, the six points $P_{1}, \ldots, P_{6}$ define a 6-tuple in $C_{2}^{(2)}$. This means that $x \in E_{2}^{(2)}$ and therefore $W=\overline{E_{2}^{(2)}}$.

Remark 3.7. How many components does the boundary of $H_{2}^{(2)}$ have? We do not know. We just know that, if $\phi\left(\Delta\left(H_{2}^{(2)}\right)\right)$ has another component then it is 
a subset of $\phi\left(\overline{2 \mathscr{A}_{2}}\right)$. For this, a cubic surface corresponding to a general point of $\phi\left(\overline{3 \mathscr{A}_{1}}\right)$ contains 3 star points and there is no line containing more than one star point.

\section{On the boundaries of $H_{4}^{(4)}$ and $H_{4}^{(6)}$}

Definition. Let $X$ be a non-singular cubic surface having two star triples with no lines in common. It follows that $X$ has a third star triple such that three corresponding star points are collinear. A such three star triples is called a StarSteiner set.

From [12], p. 289 or [13], p. 24, we have $H_{4}=H_{4}^{(4)} \cup H_{4}^{(6)} \cup H_{4}^{(9)}$ where the irreducible component $H_{4}^{(6)}\left(H_{4}^{(4)}, H_{4}^{(9)}\right.$ respectively) parametrizes non-singular cubic surfaces, each of which possesses a pair $(S, T)$ where $S$ is a Star-Steiner set and $T$ is another star triple with exactly one line (all three lines, no line respectively) in common with the lines of $S$.

\subsection{On the boundary of $H_{4}^{(6)}$}

Proposition 4.1. The set $4 \mathscr{A}_{1}$ is contained in the closure of $H_{4}^{(6)}$. Consequently, the closure of $4 \mathscr{A}_{1}$ in $\left(\boldsymbol{P}^{19}\right)^{\text {ss }}$ is an irreducible component of $\Delta H_{4}^{(6)}$.

Proof. Let $x \in 4 \mathscr{A}_{1}$. By choosing coordinates, the corresponding surface $X_{x}$ is isomorphic to the csurface of a 6-point scheme 2 , where $c(\mathscr{Q})=2 Q_{1}+$ $2 Q_{4}+Q_{2}+Q_{5}$ such that $Q_{1}, Q_{2}, Q_{3}, Q_{4}$ are in general position and the directions at $Q_{1}$ and $Q_{4}$ contain $Q_{5}$ (FIGURE $8,(\mathrm{~b})$ ). See [13], p. 96 for another configuration for $4 \mathscr{A}_{1}$ (FIGURE 8 , (a)).

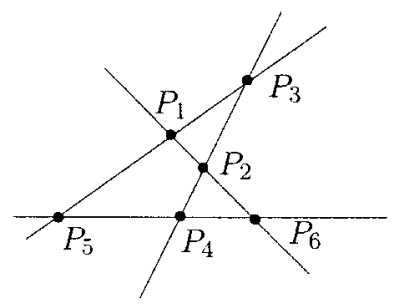

(a)

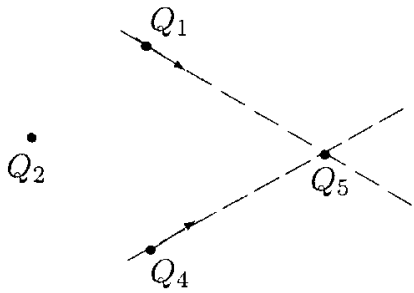

(b)

FIGURE 8. 6-point schemes giving points in $4 \mathscr{A}_{1}$

Let $C$ be the conic containing $Q_{1}, Q_{2}, Q_{4}$ and being tangent to the directions at $Q_{1}$ and $Q_{4}$. Consider a 6-point scheme $\mathscr{P}$ such that $c(\mathscr{P})=\sum_{i=1}^{6} P_{6}$ where $P_{i}=Q_{i}$ for $i \in\{1,2,4,5\}$, two points $P_{3}, P_{6}$ are contained in $C$ and $\overline{P_{1} P_{4}} \cap$ $\overline{P_{2} P_{5}} \cap \overline{P_{3} P_{6}} \neq \emptyset$ (FIGURe 9). 


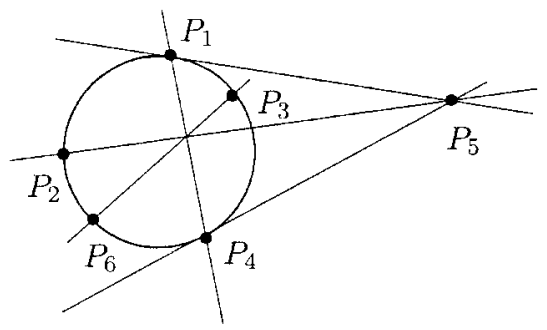

FIGURE 9. 6-point schemes giving points in $H_{4}^{(6)}$

Let $P_{3}$ and $P_{6}$ move on the conic $C$ in such a way that $\overline{P_{1} P_{4}} \cap \overline{P_{2} P_{5}} \cap$ $\overline{P_{3} P_{6}} \neq \emptyset$. We have a family of cubic surfaces in $H_{4}^{(6)}$. The 6 star points are given by $\left(\tilde{P}_{1}, \tilde{C}_{5}, \tilde{l}_{15}\right),\left(\tilde{P}_{4}, \tilde{C}_{5}, \tilde{l}_{45}\right),\left(\tilde{l}_{14}, \tilde{l}_{25}, \tilde{l}_{36}\right),\left(\tilde{C}_{4}, \tilde{P}_{2}, \tilde{l}_{24}\right), \quad\left(\tilde{P}_{5}, \tilde{C}_{2}, \tilde{l}_{25}\right)$ and $\left(\tilde{C}_{1}, \tilde{P}_{2}, \tilde{l}_{12}\right)$. When $\left(P_{3}, P_{6}\right)=\left(P_{1}, P_{4}\right)$, we get the 6-point scheme 2. This implies that $x \in \Delta H_{4}^{(6)}$.

Remark 4.2. We know that four $A_{1}$ singularities on a cubic surface corresponding to a point in $4 \mathscr{A}_{1}$ form a tetrahedron. Each edge of the tetrahedron contains one star point. Two star points on opposite edges lie on another line of $X$, which has multiplicity one. Therefore, the 6 star points of $X$ lie on a hyperplane spanned by the three lines of multiplicity 1 .

Recall that $\phi:\left(\boldsymbol{P}^{19}\right)^{s s} \rightarrow \bar{M}$ be the quotient space with respect to the action of $\operatorname{PGL}(4)$ on $\boldsymbol{P}^{19}$.

THeOREM 4.3. The set $\phi\left(\Delta H_{4}^{(6)}\right)$ consists of two points, one is the singleton $s$ and another is the image of $4 \mathscr{A}_{1}$ in $\bar{M}$.

Proof. Let $K_{4}^{(6)}$ be the set consisting of all 6-point schemes $\mathscr{P}$ in general position such that $c(\mathscr{P})=\sum_{i=1}^{6} P_{6}$ where the conic $C_{5}$ is tangent to $l_{15}$ and $l_{45}$ at $P_{1}$ and $P_{4}$ respectively; the lines $l_{14}, l_{25}$ and $l_{36}$ have one point in common (Figure 9).

The blowing-up of $\boldsymbol{P}^{2}$ at $\mathscr{P} \in K_{4}^{(6)}$ is isomorphic to a non-singular cubic surface in $H_{4}^{(6)}$. Conversely, for any $x \in H_{4}^{(6)}$, the corresponding cubic surface $X_{x}$ is isomorphic to the csurface of some 6-point scheme in $K_{4}^{(6)}$. Let $\mathscr{P} \in K_{4}^{(6)}$. Fix $P_{1}, P_{2}, P_{4}, P_{5}$ and let $P_{3}, P_{6}$ move on the conic $C_{5}$ in such a way that $\overline{P_{3} P_{6}}$ contains the intersection point of $l_{14}$ and $l_{25}$. Except for two positions determined when $\overline{P_{3} P_{6}}=l_{25}$ and $\overline{P_{3} P_{6}}=l_{14}$, the 6 points $P_{1}, \ldots, P_{6}$ define a 6-point scheme in $K_{4}^{(6)}$. This defines a surjective morphism from an open set of $\boldsymbol{P}^{1}$ to $\phi\left(H_{4}^{(6)}\right)$. This extends to a surjective morphism $\xi: \boldsymbol{P}^{1} \rightarrow \phi\left(\overline{H_{4}^{(6)}}\right)$. It is clear that when $\overline{P_{3} P_{6}}=l_{14}$, we get a point $t_{1} \in \boldsymbol{P}^{1}$ such that $\xi\left(t_{1}\right)=\phi\left(4 \mathscr{A}_{1}\right)$ (see (4.1)). When $\overline{P_{3} P_{5}}=l_{25}$, we get a point $t_{2} \in \boldsymbol{P}^{1}$. The point $\xi\left(t_{2}\right)$ corresponds to the csurface of a 6-point scheme $\mathscr{P}_{0}$ such that $c\left(\mathscr{P}_{0}\right)=2 P_{2}+P_{1}+P_{4}+P_{3}+P_{5}+P_{6}$ 
where $P_{2}, P_{3}, P_{5}$ are collinear. The csurface of 6-point scheme $\mathscr{P}_{0}$ has exactly one singular point of type $A_{2}$. Therefore $\xi\left(t_{2}\right)=s$.

Since $H_{4}^{(6)} \subset H_{2}^{(2)}$, we have:

COROLlary 4.4. The set $\phi\left(\Delta H_{2}^{(2)}\right)$ contains the singleton s.

4.2. On the boundary of $H_{4}^{(4)}$

Recall that $N=\operatorname{PGL}(4) \backslash\left(\boldsymbol{P}^{19}\right)^{s}$. We know that $\bar{M}=N \cup\{s\}$. Let

$$
\begin{aligned}
B_{4}^{(4)}=\{ & \left(P_{1}, \ldots, P_{6}, O_{1}\right) \in\left(\boldsymbol{P}^{2}\right)^{6} \mid P_{1}, P_{2}, P_{3}, P_{4} \text { are in general position; } \\
& \left.l_{12} \cap l_{34}=\left\{O_{1}\right\} ; P_{5} \in l_{13} \cap l_{24} ; P_{6} \in l_{23} \cap \overline{P_{5} O_{1}}\right\} \text { (FIgURE } 10 \text { (a)). }
\end{aligned}
$$

Note that each element in $B_{4}^{(4)}$ defines uniquely a 6-point scheme $\mathscr{P}$ such that $c(\mathscr{P})=\sum_{i=2}^{6} P_{i}$. Moreover, the csurface of $\mathscr{P}$ is isomorphic to a cubic surface with exactly three $A_{1}$ singularities (see [13], pp. 74-75). Let $D_{4}^{(4)}$ be the subset of $\boldsymbol{P}^{19}$ consisting of all points such that each corresponding cubic surface is isomorphic to the csurface of a 6-point scheme determined by some element in $B_{4}^{(4)}$. We see that $D_{4}^{(4)} \subset 3 \mathscr{A}_{1}$.

Let

$$
\begin{aligned}
C_{4}^{(4)}=\{ & \left(P_{1}, \ldots, P_{6}, O_{1}, O_{2}\right) \in\left(\boldsymbol{P}^{2}\right)^{6} \mid P_{1}, P_{2}, P_{3}, P_{4} \text { are in general position; } \\
& \left.l_{12} \cap l_{34} \cap l_{56}=\left\{O_{1}\right\} ; l_{13} \cap l_{24} \cap l_{56}=\left\{O_{2}\right\} ; l_{12} \cap l_{36} \cap l_{45} \neq \emptyset ; P_{5} \in l_{23}\right\}
\end{aligned}
$$

(Figure $10(\mathrm{~b})$ ). Each element in $C_{4}^{(4)}$ defines uniquely a 6-point scheme $\mathscr{P}$ such that $c(\mathscr{P})=\sum_{i=1}^{6} P_{i}$. Moreover, the csurface of $\mathscr{P}$ is isomorphic to a cubic surface with exactly one $A_{1}$ singularity. Let $E_{4}^{(4)}$ be the subset of $\boldsymbol{P}^{19}$ consisting of all points such that each corresponding cubic surface is isomorphic to the csurface of a 6-point scheme determined by some element in $C_{4}^{(4)}$. We see that $E_{4}^{(4)} \subset \mathscr{A}_{1}$.

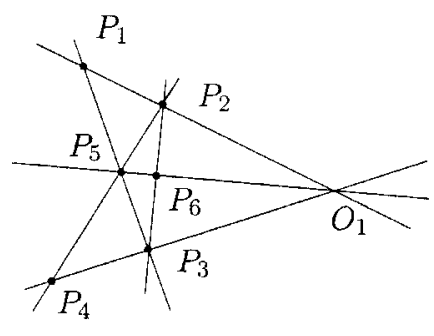

(a)

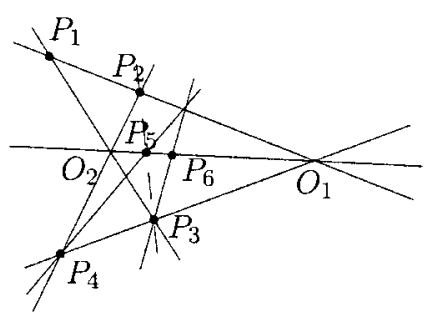

(b)

FIGURE 10. 6-point schemes of $B_{4}^{(4)}$ and $C_{4}^{(4)}$, respectively

Proposition 4.5. The closures of $D_{4}^{(4)}$ and $E_{4}^{(4)}$ in $\left(\boldsymbol{P}^{19}\right)^{s s}$ are irreducible components of $\Delta H_{4}^{(4)}$. 
Proof. Let $K_{0}=\left\{\left(P_{1}, P_{2}, P_{3}, P_{4}\right) \in\left(\boldsymbol{P}^{2}\right)^{4} \mid P_{1}, P_{2}, P_{3}, P_{4}\right.$ are in general position $\}$. Consider the projection $p: B_{4}^{(4)} \rightarrow K_{0}$. We see that $p$ is an isomorphism. So $B_{4}^{(4)}$ is irreducible. Similarly, the projection $C_{4}^{(4)} \rightarrow K_{0}$ is an isomorphism. So the set $C_{4}^{(4)}$ is irreducible. By a similar argument as used in the proof of (3.2), we see that $D_{4}^{(4)}$ and $E_{4}^{(4)}$ are irreducible.

Let $x \in D_{4}^{(4)}$. By definition, the surface $X_{x}$ is isomorphic to the csurface of a 6-point scheme $\mathscr{P}$ determined by an element $\left(P_{1}, \ldots, P_{6}, O_{1}\right)$ of $B_{4}^{(4)}$. Fix 4 points $P_{1}, P_{2}, P_{3}, P_{4}$. Let $l_{13} \cap l_{24}=\left\{O_{2}\right\}$. Let $P_{5}^{t}, P_{6}^{t}$ move on the line $\overline{O_{1} O_{2}}$ in such a way that $l_{45} \cap l_{36} \cap l_{12} \neq \emptyset$. Except for a finite number of positions of $\left(P_{5}^{t}, P_{6}^{t}\right)$, the 6 points $P_{1}, \ldots, P_{4}, P_{5}^{t}, P_{6}^{t}$ form a 6-point scheme $\mathscr{P}$ such that the csurface of $\mathscr{P}$ is isomorphic to a cubic surface in $H_{4}^{(4)}([12]$, p. 289). The four star points are given by $\left(\tilde{l}_{12}, \tilde{l}_{34}, \tilde{l}_{56}\right),\left(\tilde{l}_{12}, \tilde{l}_{36}, \tilde{l}_{45}\right),\left(\tilde{l}_{13}, \tilde{l}_{24}, \tilde{l}_{56}\right)$ and $\left(\tilde{l}_{34}, \tilde{l}_{15}, \tilde{l}_{26}\right)$. We obtain a family in $H_{4}^{(4)}$. It is clear that $x$ is a specialization position of this family.

Similarly, if $x \in E_{4}^{(4)}$, we consider a family defined as above. The point $x$ is a specialization position which is determined when $l_{23} \cap \overline{O_{1} O_{2}}=\left\{P_{5}\right\}$.

Remark 4.6. It is not clear that if the boundary of $H_{4}^{(4)}$ in $\bar{M}$ contains the singleton. However, we can prove the following corollary.

COROLlary 4.7. The set $\phi\left(\Delta H_{4}^{(4)}\right) \cap N$ consists of two points which are the image of $\overline{D_{4}^{(4)}}$ and $\overline{E_{4}^{(4)}}$.

Proof. See [13], 3.4.24.

\section{Acknowledgement}

Some results in this paper were obtained during Ph.D. study of the author under a fellowship of Utrecht University, The Netherlands and also contained in Chapter 3 of his thesis [13]. The author would like to express deep gratitude to his Ph.D. Supervisor, Prof. Dr. F. Oort for careful guidance and endless support. This paper is improved and completed when the author is supported by the JSPS Postdoctoral Fellowship. The author also thanks his host researcher, Prof. Dr. M. Oka, Tokyo Metropolitan University, Japan for fruitful discussions and valuable comments.

\section{REFERENCES}

[1] V. I. ARNOL'D, Normal forms for functions near degenerate critical points, the Weyl groups of $A_{k}, D_{k}, E_{k}$ and Lagrangian singularities, Funk. Anal. Appl. 6 (1972), 254-272.

[2] V. I. ARNOL'D, Normal forms of functions near degenerate critical points, Russian Math. Surveys, 29 (2) (1974), 11-49.

[ 3 ] J. W. Bruce, A stratification of the space of cubic sufaces, Math Proc. Camb. Phil. Soc. 87 (1980), 427-441. 
[ 4 ] J. W. Bruce, C. T. C. Wall, On the classification of cubic surfaces, J. London Math. Soc. (2) 19 (1979), 245-256.

[5] M. Brundu, A. Logar, Parametrization of the orbits of cubic surfaces, Transformation Groups, 3 (4) (1998), 1-31.

[6] A. Cayley, A memoir on cubic surfaces, Phil. Trans. Roy. Soc. 159 (1869), 231-326.

[7] R. Hartshorne, Algebraic Geometry, Grad. Texts in Math. 52, Springer-Verlag 1977.

[ 8 ] D. Mumford, J. Fogarty, F. Kirwan, Geometric Invariant Theory (Third Enlarged Edition), Springer-Verlag 1994.

[9] D. Mumford, Stability of projective varieties, Enseign. Math, 23 (1977), 39-110.

[10] I. Naruki, Cross ratio varieties as a moduli space of cubic surfaces, Proc. London Math. Soc. (3), 45 (1982), 1-80.

[11] P. E. Newstead, Lectures on Introduction to Moduli Problems and Orbit Spaces, Tata Inst. Lecture Notes, Springer-Verlag 1978.

[12] T. C. NGUYeN, Non-singular cubic surfaces with star points, preprint nr. 1082, Department of Mathematics, Utrecht University (12/1998), Vietnam Journal of Mathematics, 29:3 (2001), 287-292.

[13] T. C. NGuyen, Star points on cubic surfaces, Doctoral Thesis, Utrecht University, The Netherlands (11/2000), ISBN: 90-393-2575-8, http://www.library.uu.nl/digiarchief/dip/diss/ 1933178/inhoud.htm

Department of Mathematics

TOKyo Metropolitan University

Minami-OHSAWa 1-1, HachioJI-SHI

TOKYO 192-0397

JAPAN

e-mail: chanhtu@math.metro-u.ac.jp 